\title{
Active polymer translocation in the three-dimensional domain
}

\author{
A. Fiasconaro, ${ }^{1,2,3, *}$ J. J. Mazo, ${ }^{1,2}$ and F. Falo ${ }^{1,4}$ \\ ${ }^{1}$ Departamento de Física de la Materia Condensada, Universidad de Zaragoza, 50009 Zaragoza, Spain \\ ${ }^{2}$ Instituto de Ciencia de Materiales de Aragón, C.S.I.C.-Universidad de Zaragoza, 50009 Zaragoza, Spain \\ ${ }^{3}$ School of Mathematical Sciences, Queen Mary University of London, Mile End Road, London E1 4NS, United Kingdom \\ ${ }^{4}$ Instituto de Biocomputación y Física de Sistemas Complejos, Universidad de Zaragoza, 50018 Zaragoza, Spain
}

(Received 7 July 2014; revised manuscript received 7 November 2014; published 11 February 2015)

\begin{abstract}
In this work we study the translocation process of a polymer through a nanochannel where a time dependent force is acting. Two conceptually different types of driving are used: a deterministic sinusoidal one and a random telegraph noise force. The mean translocation time presents interesting resonant minima as a function of the frequency of the external driving. For the computed sizes, the translocation time scales with the polymer length according to a power law with the same exponent for almost all the frequencies of the two driving forces. The dependence of the translocation time with the polymer rigidity, which accounts for the persistence length of the molecule, shows a different low frequency dependence for the two drivings.
\end{abstract}

DOI: 10.1103/PhysRevE.91.022113 PACS number(s): 05.40.-a, 87.15.A-, 87.10.-e, 36.20.-r

\section{INTRODUCTION}

The study of polymer translocation through natural and/or artificial nanopores is important for understanding many biological processes and for developing a family of new devices working at the nanoscale [1-3]. In biology, interest is focused in the study of DNA, RNA, and protein translocation through cell membranes and nuclear pores, DNA injection by phage viruses, cell drug delivery, DNA sequencing, etc., thus resulting a most interesting topic which involves a broad phenomenology. The passage of polymers thorough the nanopores is also important in many chemical and industrial processes. In this context, nanotechnological applications try to emulate the complex biological processes involved in the translocation problem [4-6].

In spite of the many recent advances, our understanding of polymer translocation at the nanoscale is still limited and controversial. Recently, some unified visions of the translocation physics have been proposed $[7,8]$. Motivated by many experimental results, different models have been introduced to describe and study in a simple way this and related problems $[9,10]$. In some models a single barrier potential is introduced, eventually depending on time [11-13]; in others, stochastic and ratchetlike forces and potentials are used $[14,15]$.

In some cases the transport phenomena involve not only translocation through passive channels, but also molecular motors (or active nanopores), whose complex action is attracting an increasing interest $[16,17]$. Thus, various models for translocation through active pores study periodic sinusoidal pore actuation [18-21], stochastic random telegraph noise (RTN) [21,22], or a dichotomous ATP-based motor noise $[23,24]$. These different models, mostly based on modifications of the Rouse chain [25] in the one-dimensional (1D) or $2 \mathrm{D}$ domains, have revealed various (sometimes similar) behaviors in the translocation times of the polymer as a function of the nature and the frequency of the time dependent driving, as well as with the dimensionality of the system. In

\footnotetext{
*A.Fiasconaro@qmul.ac.uk
}

addition, recent simulations and experiments suggest that the translocation process significantly depends on the size and flexibility of the chain and on the interactions between the polymers and the pores [26-29].

Aimed by all these results, we consider here the case of a linear polymer molecule in the 3D domain driven by two different time dependent forces in the nanopore: a RTN and a sinusoidal force. We especially focus on the study of the mean translocation time $\tau$ of the polymer as a function of the frequency of the external driving for different lengths and flexibilities of the chain.

Sinusoidal driving is the simplest approach to a periodic actuation at the pore. Beyond its paradigmatic interest, its study is motivated in a nanotechnological level in a context of manmade active nanopores. The dichotomous driving makes sense both in the nanotechnological and in the biophysical contexts. In the first case the passage of a polymer can be induced through a graphene pore or solid state channeling [30,31] by applying a dichotomous force between the two sides of the layer. In the second case, the model can describe the translocation of a linear molecule through a cell membrane gate having a chemical potential difference between its two sides. Then the driving is induced by the typical open-close mechanism of the pore, and follows the purely dichotomous switching largely used in the literature $[15,32,33]$. The application of a dichotomous force during the translocation of a DNA through a nanopore can also be relevant for characterizing the nucleotides which cross the pore, thus representing a possible DNA sequencing method [27,34].

The 3D polymer considered here is an extension of the system studied in our previous works [18,22,23]. It consists of $N$ beads connected by elastic bonds, where we added the bending features typical of a wormlike-chain model. Excluded volume effects and polymer-pore-membrane interactions have been taken into account by means of a Lennard-Jones potential.

We study two observables of the system for the different actuation frequencies: the translocation probability $P_{i n}$ and the mean translocation time $\tau$. The results of the computer simulations for different polymer sizes and different persistence lengths will be shown. Important unexpected discrepancies in the frequency behavior between the two drivings are found. 
For the sinusoidal force the mean translocation time as a function of the frequency shows some oscillations, similar to those found in the simpler $1 \mathrm{D}$ and $2 \mathrm{D}$ cases $[18,21]$. On the contrary, the RTN actuation shows different qualitative behavior in the translocation time (TT) at low and high chain flexibility at the low frequency regime. A resonant activation (RA) phenomenon [35-37], i.e., the presence of a minimum in the TT as a function of the driving frequency, is observed in both drivings. The scaling properties of $\tau$ with the polymer size follow a power law trend, with a different exponent from that one found in the $1 \mathrm{D}$ case, but the same in that the chain is driven by a constant force. The analysis of the translocation of the polymer for different values of the flexibility parameter shows significant differences in the polymer translocation probability at low frequency with sinusoidal and RTN driving.

We first present the model of the polymer and the equation of motion. Then we show the results of the simulations with the sinusoidal forces (Sec. III A) and RTN driving (Sec. III B). The conclusions end the paper.

\section{THE MODEL}

We model the linear polymer molecule as a chain formed by $N$ identical monomers moving in 3D. We use a modified Rouse model [25], which includes polymer elasticity, bending energy, excluded volume effects and interaction with the membrane and the pore. The elastic potential energy is given by

$$
V_{\mathrm{el}}\left(d_{i}\right)=\frac{k_{e}}{2} \sum_{i=1}^{N}\left(d_{i}-l_{0}\right)^{2}
$$

where $k_{e}$ is the elastic parameter, $\mathbf{r}_{i}$ is the position of the $i$ th particle, $d_{i}=\left|\mathbf{d}_{i}\right|=\left|\mathbf{r}_{i+1}-\mathbf{r}_{i}\right|$ is the distance between the monomers $i$ and $i+1$, and $l_{0}$ is the equilibrium distance between adjacent monomers.

The model takes into account the bending energy of the chain with a term given by

$$
V_{\mathrm{ben}}\left(\theta_{i}\right)=\frac{k_{b}}{2} \sum_{i=1}^{N}\left[1-\cos \left(\theta_{i}-\theta_{0}\right)\right],
$$

where $k_{b}$ is the bending elastic constant, $\theta_{i}$ is the angle between the link $\mathbf{d}_{i+1}$ and the link $\mathbf{d}_{i}$, and $\theta_{0}$ is the equilibrium angle, $\theta_{0}=0$ in our case.

To consider excluded volume effects between the monomers, a repulsive only Lennard-Jones potential has been taken into account,

$$
V_{\mathrm{LJ}}(r)=4 \epsilon\left[\left(\frac{\sigma}{r}\right)^{12}-\left(\frac{\sigma}{r}\right)^{6}\right],
$$

for $r \leqslant 2^{1 / 6} \sigma$, and zero otherwise.

The dynamics of every monomer of the chain is obtained by the overdamped equation of motion

$$
\begin{aligned}
m \Gamma \dot{\mathbf{r}}_{i}= & -\nabla_{i} V_{\mathrm{el}}\left(d_{i}\right)-\nabla_{i} V_{\mathrm{ben}}\left(\theta_{i}\right)-\nabla_{i} V_{\mathrm{LJ}}\left(d_{i}\right) \\
& +F_{\mathrm{drv}, i} \mathbf{i}+\mathbf{F}_{\mathrm{sp}, i}+\sqrt{2 m \Gamma k_{B} T} \vec{\xi}_{i}(t),
\end{aligned}
$$

where $\Gamma$ and $m$ are the damping parameter and mass of each monomer, respectively. $\vec{\xi}_{i}(t)$ stands for the Gaussian uncor-

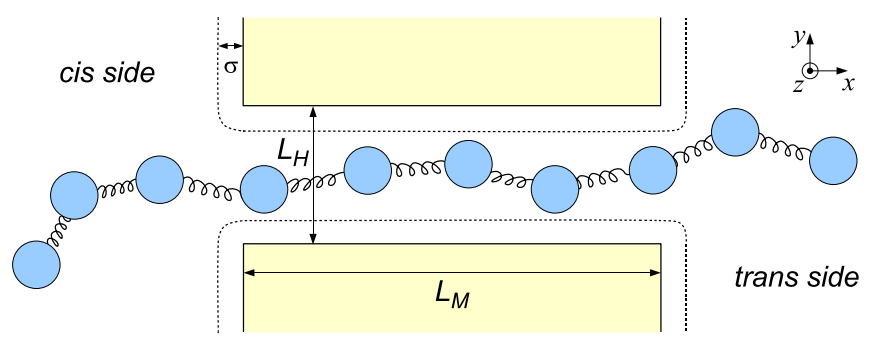

FIG. 1. (Color online) Section of the polymer translocating through a nanopore in $3 \mathrm{D}$. The pore has a square section of width $L_{H}$ and length $L_{M}$. Inside, the pore acts a driving force in the $x$ direction, which pushes the polymer toward the trans side of the membrane. The walls of the membrane repulse uniformly the chain inside a characteristic distance $\sigma$.

related thermal fluctuation and follows the usual statistical properties $\left\langle\xi_{i, \alpha}(t)\right\rangle=0$ and $\left\langle\xi_{i, \alpha}(t) \xi_{j, \beta}\left(t^{\prime}\right)\right\rangle=\delta_{i j} \delta_{\alpha, \beta} \delta\left(t^{\prime}-t\right)$, with $i=1, \ldots, N$, and $\alpha, \beta=x, y, z$. The operator $\nabla_{i}=$ $\partial / \partial x_{i} \mathbf{i}+\partial / \partial y_{i} \mathbf{j}+\partial / \partial z_{i} \mathbf{k}$.

The two explicit forces in Eq. (4) account for the driving forces over the particles inside the pore $F_{\mathrm{drv}}$ and the chain-membrane and chain-pore spatial constraints $\mathbf{F}_{s p}$. This interaction force is also modeled by using the same repulsive Lennard-Jones potential described in Eq. (3), with the same potential parameters. It takes place uniformly and perpendicularly to all the planes that define both the membrane and the pore channel, the latter modeled as a square prism of base $L_{M}$ and length $L_{H}$ (see Fig. 1). $F_{\text {drv }}$ only affects the particles inside the pore.

To complete this section, we list the parameters used in the simulations. The rest distance between adjacent monomers is $l_{0}=1$, and we fix $k_{e}=1600$ to be large enough to keep the bonds rigid. The temperature used is $k_{B} T=0.1$. The LennardJones parameters are $\epsilon=0.3$ and $\sigma=0.8$.

Following [21], we can set $\epsilon, m$, and $l_{0}$ as the energy, mass, and length units, respectively. This choice determines a Lennard-Jones time scale given by $t_{\mathrm{LJ}}=\left(m l_{0}^{2} / \epsilon\right)^{1 / 2}$. However, as the dynamics we propose is overdamped, the time scale that normalize the equation of motion (4) is $t_{\mathrm{OD}}=\Gamma t_{\mathrm{LJ}}{ }^{2}$, thus depending on the damping parameter. This time unit also determines the frequency unit. To set some values, let us consider a DNA molecule at room temperature $\left(k_{B} T=\right.$ $4.1 \mathrm{pN} \mathrm{nm})$ and the simple case with $k_{b}=0$. In our simulation, $k_{B} T=\epsilon / 3$ is fixed and we set $l_{0}=1.25 \sigma=1.875 \mathrm{~nm}$ and $m=936$ amu [21]. With these values we have $t_{\mathrm{LJ}} \approx 6,7 \mathrm{ps}$. The force unit is given by $\epsilon / l_{0}=6.47 \mathrm{pN}$. An estimation for the kinetic damping is $\Gamma \approx 1.610^{13} \mathrm{~s}^{-1}$; thus, $t_{\mathrm{OD}} \approx 720 \mathrm{ps}$. Note that this election is arbitrary and depends on the particular system we are trying to simulate (for instance, another value for the length unit is given in a similar model in [38]).

\section{RESULTS}

We are interested in characterizing the translocation process of the polymer through the pore. We present below results for the mean value of the TT of the chain for different bending parameters and different polymer lengths. Then a clear definition of the computed mean TT is needed: The simulations start with five monomers at one end of the polymer inside the 
pore and the others linearly ordered and sited at the equilibrium distance in the cis side. During a thermalization time $t_{t}=1000$ a.u. the chain evolves under the action of thermal fluctuations with the position of the five monomers inside the pore kept fixed. After that transient time, the restriction over the first five monomers is removed, and the evolution of the chain under the dynamics given by Eqs. (4) is monitored. In some cases, the polymer moves backwards into the cis region and does not translocate. These cases are not taken into account in the statistics. Whenever the polymer crosses the membrane, the simulation ends when the last monomer of the chain enters in the pore, which defines a translocation event, and the time from the simulation start gives the TT. Later we average over many translocation processes to get $\tau_{N}$. With this definition we reduce possible polymer size effects due to the pull and push of the chain, whereas a few monomers lie inside the motor. Other definitions are reasonable [21,39]. In a few specific cases in this work, another TT is defined as the time for the center of mass (c.m.) of the chain to reach the trans side. The average of this quantity over the simulations defines $\tau_{c . m}$. As we also see, no qualitative differences between the two measures appear.

The geometrical values used in the simulations for the square pore are $L_{H}=2, L_{M}=5.5$. The combination of values for $L_{M}$ and $L_{H}$ guarantees that the polymer is maintained approximately straight and the monomers sequentially ordered inside the pore, without any knot or monomer crossing. The suitable choice of the bending constant $k_{b}$ opens also the possibility to study the TT for different persistence lengths $L_{p}$ of the chain, which basically gives the stiffness of the polymer (i.e., its resistance to bend). For our model $L_{p}=k_{b} / k_{B} T$. Thus, for example, $L_{p}=5$ (in units of $l_{0}$ ) for $k_{b}=0.5$, a value we use below.

For every set of parameters, a number of numerical experiments $N_{g}$ has been simulated. From them a number $N_{t}=2000$ successful translocations have been reached and the mean TT $\tau$ over $N_{t}$ has been computed. From these values, we define the translocation probability $P_{i n}$ as the ratio $N_{t} / N_{g}$. To finish, since we deal with time dependent forces, an averaging over their initial values is also performed: In practice, the initial phase of the forces is considered as a random variable with a uniform distribution of all its possible values.

As we see, depending on the frequency, three regions can be distinguished: The high frequency region, where the fluctuations are much faster than the typical TT; the low frequency region, where the fluctuations are much slower than the typical TT and the large intermediate frequency region where the phenomenology becomes rich and interesting.

\section{A. Sinusoidal driving}

The sinusoidal force used to drive the system has the form

$$
F_{\mathrm{drv}}(x, t)= \begin{cases}F_{0}[1-\cos (2 \pi v t+\phi)], & x \in\left[0, L_{M}\right] \\ 0, & \text { otherwise }\end{cases}
$$

with the value $F_{0}=0.3$ adopted in all the simulations and $\phi$ the randomly chosen initial phase.

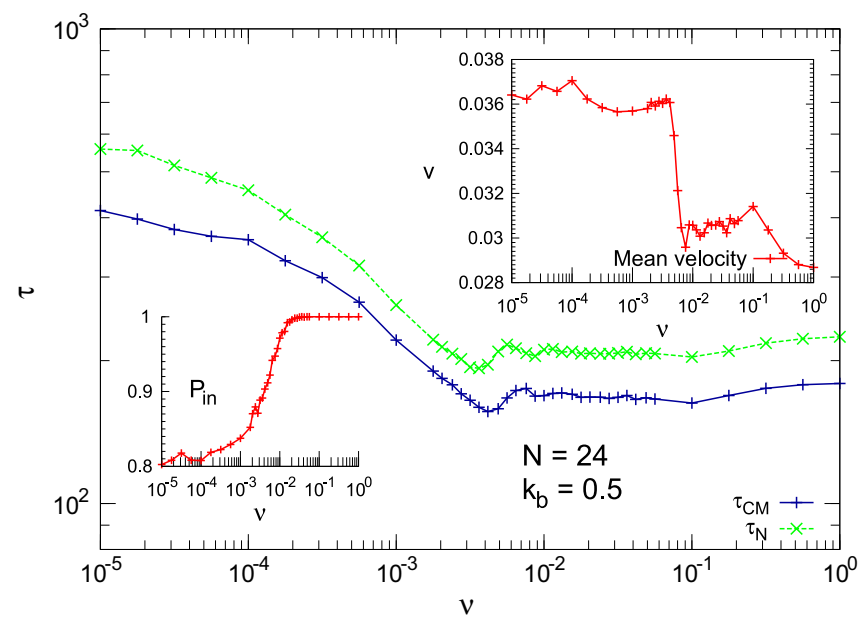

FIG. 2. (Color online) Mean TTs $\tau_{c . m .}$ and $\tau_{N}$ as a function of $v$ for the polymer with $N=24$, and bending $k_{b}=0.5$ under sinusoidal driving. The top inset shows the mean velocity of the translocation (c.m.). The left inset shows the translocation probability $P_{i n}$.

\section{Translocation time}

Figure 2 shows the mean TT as a function of the driving frequency for a polymer with $N=24$ and $k_{b}=0.5$. The results are shown for the two definitions of TT given above. The three expected frequency regions are found. For high frequencies the change in the external force is much faster than the time scales involved in the polymer dynamics, and the monomers in the pore experience an apparently constant $F_{\mathrm{drv}}=0.3$ force. For smaller frequencies we enter into a more complex and dynamically interesting region characterized by a minimum in the TT (or a peak in the polymer velocity; see top inset) and a series of oscillations. In this region the driving period is of the same order of magnitude of the typical TT.

The appearance of a large minimum region in the $\tau$ curve, resembles the RA phenomenon, where an optimum range of frequencies for a thermal escape event is found. RA is essentially due to a synchronization of two time scales (driving period and $\tau$ in our problem) and can be also observed without a potential barrier to overcome [18].

Frequencies below the resonant region define the low frequency region, where $\tau$ increases and saturates at very small frequency values. For such limit frequencies the pore basically exerts a constant force established by the initial random phase. Thus, a wide distribution of TTs is expected, as well as an important number of negative translocation events: The repulsive interaction of the chain with the membrane walls, together with the dynamical polymer configuration, results in an average force than tends to avoid the polymer translocation. This generates an entropic barrier effect [26], which opposes the polymer translocation, that we measure indirectly by evaluating the a posteriorioverall translocation probability $P_{i n}$ for the polymer to cross into the trans region. As we see more extensively below in the comment of Fig. 11 in the discussion section, at low values of constant forces the translocation probability becomes very small. Then events starting with a large enough initial force (determined by the random phase $\phi$ ) will be able, on average, to move the 
polymer through the pore, but not the ones with low (or null) initial force. The bottom inset of Fig. 2 shows that, at low frequencies, $80 \%$ of the simulated events translocate for the sinusoidal case. These entropic barrier effects can be evaluated following the theory presented by Rowghanian and Grosberg in Ref. [40], where a careful analysis of the conformational and orientational entropic contributions for the polymer close to the pore is presented.

The observed behavior of $\tau$ is qualitatively similar to that found in a similar model for 1D chains where a minimum and a series of oscillations is reported [18]. These oscillations arise from the synchronization between the period of the force and the very time spent by the polymer in the translocation process. Their presence has been also reported by other works with oscillating driving [19,21].

Regarding the 2D case studied in [21], differences are observed at low frequencies. There, using a freely jointed chain (FJC) model, a decrease of $\tau$ for decreasing frequencies is reported. The FJC model corresponds to $k_{b}=0$ in our study, a case displayed in Fig. 5, where the same qualitative behavior for all values of $k_{b}$ is found. This discrepancy is caused by the very different translocation events at low frequencies. Our initial condition (five monomers inside the pore) ensures a high translocation probability. Then slow events are observable at low frequencies. The dimensionality also plays a role by increasing the TTs. In [21] the initial condition is different (one monomer inside the pore), so only a small number of trials translocate, and they do it with small TTs. More precisely, the successful translocation rate is around $80 \%$ at low frequency. This implies that most of the initial phases contribute to the translocation, also those with initial small forces, i.e., initial phase close to zero. These low values of the initial force give slow events that contribute by increasing the mean TT. This means that for low frequencies, the simulation is essentially averaging the times over the statistics of translocation mainly given by a range of forces between $F_{r}$ and $F_{0}$, where $F_{r}<F_{0}$ is the minimum amount of force which can overcome the entropic retracting force in almost all the trials.

The probability distribution function of the TTs is shown in Fig. 3 for several values of the frequency of the driving, namely, $v=10^{-1}, 10^{-1.75}, 10^{-2.2}, 10^{-2.4}, 10^{-2.5}$, and $10^{-4.5}$. The first and the last values correspond, respectively, to the high frequency limit, where an almost Gaussian TT distribution is found, and the low frequency limit, which shows a very asymmetric and wide distribution, with very short and very long TT events. The other ones, corresponding to the intermediate frequency region, reveal the rich resonant phenomenology that is related to the starting phase of the oscillating driving force [21].

\section{Scaling with $N$}

An interesting issue is the study of the scaling of the TT of the polymer with its size. Figure 4 shows $\tau(v)$ curves for different values of $N$. As shown in the top inset, all the curves are qualitatively similar and $\tau$ increases with the polymer size. In fact, for the range of $n$ values we use, we find that the mean TT scales as

$$
\tau \propto L^{\alpha}
$$
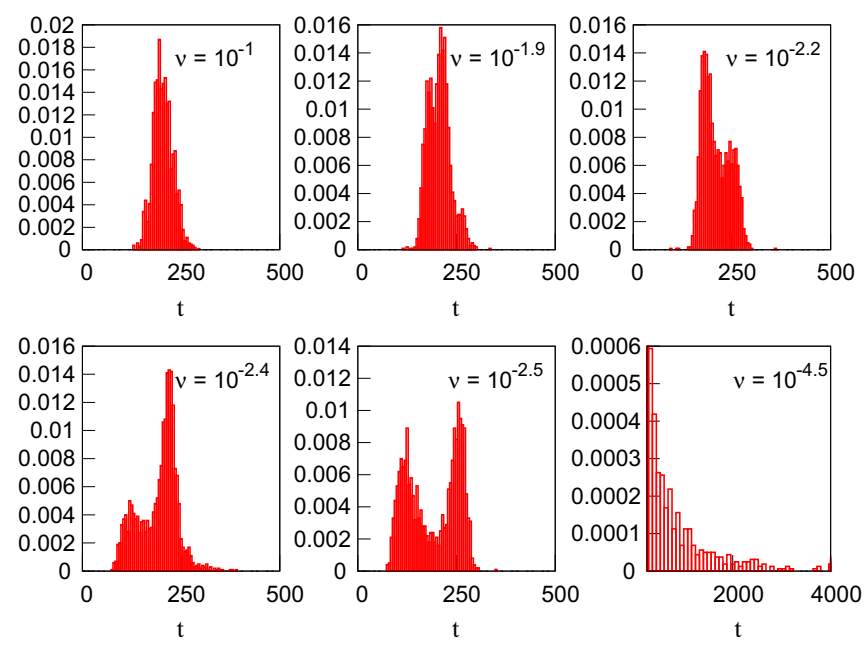

FIG. 3. (Color online) Probability distribution of the TTs at different driving frequencies. The parameters are the same as in Fig. 2.

with $\alpha=1.6$, as in the constant forced case, and $L=$ $l_{0}(N-1)$.

It is interesting to note that the exponent here is smaller than the one found in the $1 \mathrm{D}$ case [18], where $\alpha=2.0$. As expected, the dimensionality does affect the scaling properties of the chain. Importantly, we see that the same exponent is found for RTN, the other driving studied in this paper. It can be shown that the TT of a polymer scales as $\tau \propto L^{1+v_{F}}$, with $\nu_{F}$ the Flory coefficient. $v_{F}=0.5$ for an ideal polymer, $v_{F} \simeq 0.6$ if we consider the excluded volume contribution (no bending), and $v_{F}=1$ for a rod (rigid chain) $[7,41,42]$. The value we find here, $v_{F}=0.6$, for a bending of $k_{b}=0.5$ lies in one extreme of this interval [43].

Figure 4 also shows the translocation probability $P_{i n}$ for different $N$. We notice that for large polymers $P_{i n}$ decreases at low frequencies: In a small percentage of the cases the polymer stays in the cis region and does not translocate. However, at a

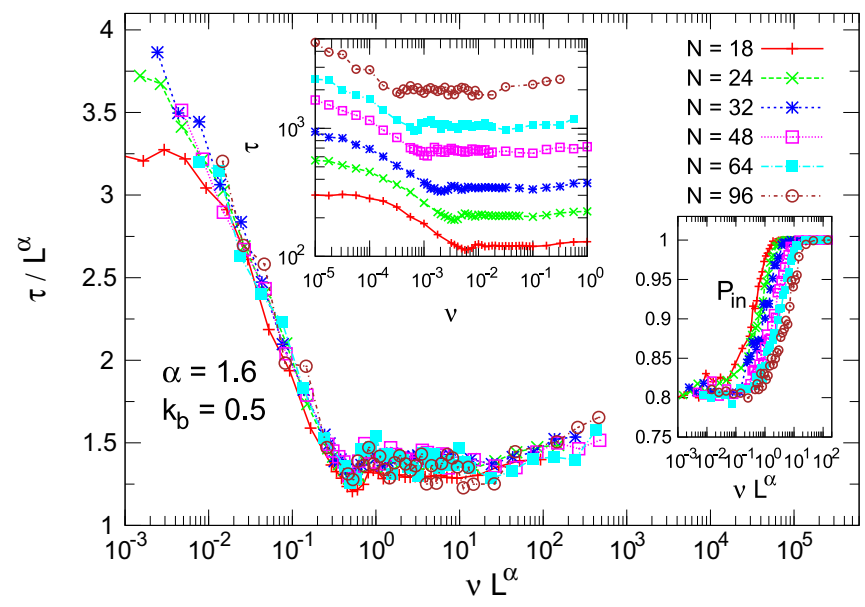

FIG. 4. (Color online) Scaled mean TTs $\left(\tau_{N}\right)$ for different values of the polymer length in the case of sinusoidal driving with bending $k_{b}=0.5$. (Top inset) Unscaled mean TT. (Right inset) Translocation probability $P_{i n}$ for the different polymer lengths. 


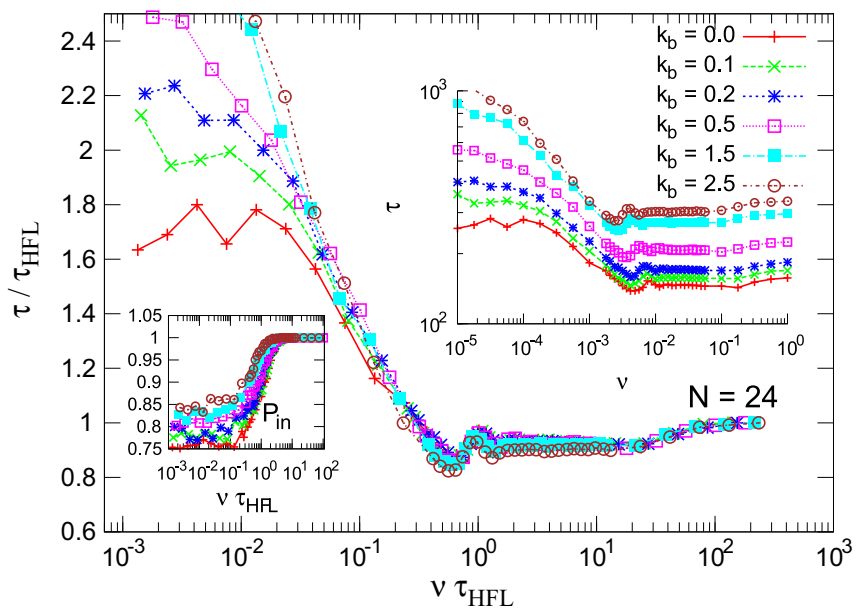

FIG. 5. (Color online) Normalized mean TT $\left(\tau_{N}\right)$ of a polymer with $N=24$ monomers for different values of the bending parameter $k_{b}$ in the case of sinusoidal driving. (Right inset) Non-normalized curves. (Left inset) Translocation probability.

given frequency, which depends on the length $N$, this fraction rapidly increases to 1 .

\section{The role of the bending parameter}

Our model of the polymer is basically a discrete wormlikechain (WLC) model, with extensible bonds. In order to study the translocation behavior of the polymer at different values of the persistence length $L_{p}$, we have performed simulations for different values of the bending parameter $k_{b}$ from $k_{b}=0$ (FJC model) to high $k_{b}$ values (extensible rigid chain). The response of the mean TT of the polymer at different bending parameters is shown in Fig. 5 (top inset). The increase of the $\tau$ with increasing values of the bending parameter is due not only to the different dynamics for the various values of $k_{b}$, but also to the different polymer configuration after the thermalization time. In fact, being the last five monomers fixed inside the channel during thermalization, at high bending the polymer remains straight, but at low bending the polymer contracts. Thus, the starting position of the c.m. in the two cases is different and, consequently, the crossing time is lower for high bending. The presence of the interactions with the walls goes in an opposite direction, but its effect is evidently smaller compared to this geometric fact. A similar argument is given in Adhikari and Bhattacharya [44], reporting Kantor and Kardar [45].

For a better comparison, the main figure shows $\tau$ normalized by the high frequency limit values $\tau_{\mathrm{HFL}}$. Then all curves collapse to a single one in the intermediate and high frequency regions of the system.

The left inset of Fig. 5 shows $P_{i n}$ for the different $k_{b}$ values. We notice that the different bending values give different $P_{i n}$ at low frequencies: Stiffer chains (higher $k_{b}$ values) show a translocation probability greater than that one of soft chains (low $k_{b}$ values). In this case, in fact, the interaction with the repulsive wall of the component of the chain is higher due to the wider movement of the chain. Nevertheless, such behavior does not give any qualitative difference in the mean TT curves.

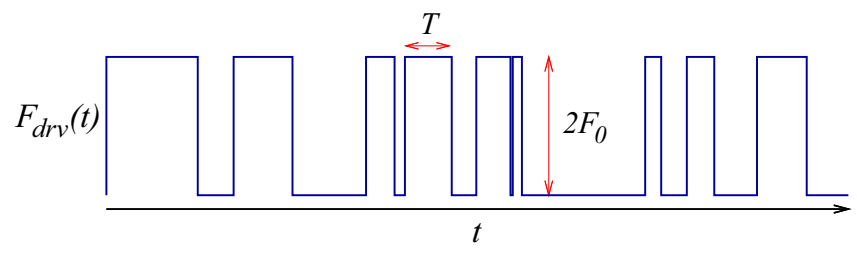

FIG. 6. (Color online) Time diagram of the RTN force.

\section{B. Random telegraph noise driving}

We present now our results for a very different type of driving, the RTN, where the force switches between 0 and $2 F_{0}$ with a given time scale $T$; see Fig. 6 . For this dichotomous noise,

$$
\begin{aligned}
\left\langle F_{\mathrm{drv}}(t)\right\rangle & =F_{0}, \quad \text { and } \\
\left\langle F_{\mathrm{drv}}(t) F_{\mathrm{drv}}\left(t^{\prime}\right)\right\rangle & =4 F_{0}^{2}\left(1+e^{-2\left(t-t^{\prime}\right) / T}\right),
\end{aligned}
$$

with $T=1 / v$ the mean residence time in each one of the two states. Regarding the spatial dependence,

$$
F_{\text {drv }}(x)=\left\{\begin{array}{lr}
\left(0,2 F_{0}\right), & x \in\left[0, L_{M}\right], \\
0, & \text { otherwise }
\end{array}\right.
$$

As in the sinusoidal case, we use $F_{0}=0.3$.

\section{Translocation time}

The mean TT of the polymer as a function of $v$ is shown in Fig. $7\left(N=24\right.$ and $\left.k_{b}=0.5\right)$. We see that $\tau$ changes smoothly from the low frequency value to the high frequency ones (note that $\tau_{H F L}$ is the same as that one in the sinusoidal case), showing a wide minimum for an intermediate frequency (more clearly visible in Fig. 9).

Although important differences with respect to the sinusoidal case appear in the intermediate frequency region, the presence of this minimum is well visible and appears as the synchronization of two defined time scales, the mean TT of the polymer and the RTN mean waiting time scale, so giving a typical RA behavior.

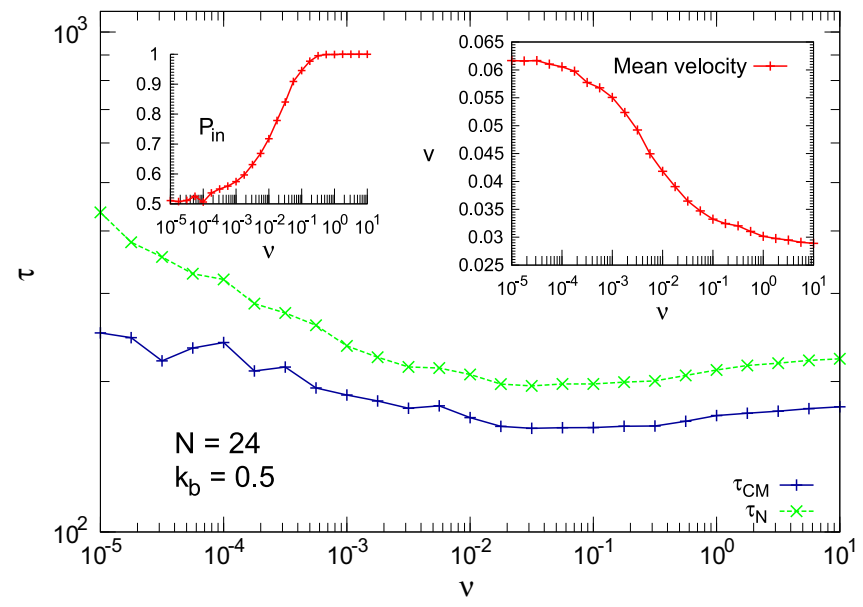

FIG. 7. (Color online) Mean TTs $\tau_{c . m}$. and $\tau_{N}$ of the polymer under RTN driving. The parameters are $N=24, k_{b}=0.5$. The right inset shows the mean velocity. The left inset shows the polymer translocation probability $P_{i n}$. 

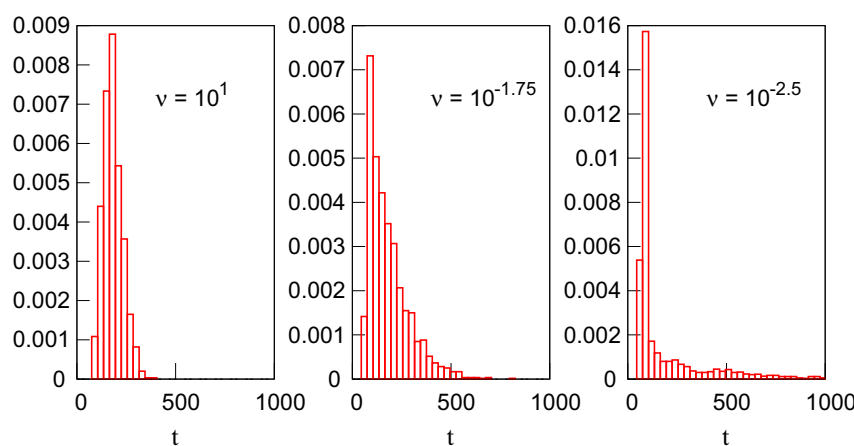

FIG. 8. (Color online) Probability distribution of the polymer TTs at different frequencies of the driving $v=1 / T$. The statistics refer to the values of Fig. 7 .

Figure 7 also shows the translocation probability of the polymer and the mean translocation velocity. We see that the $v$ curve shows a saturating maximum at the low frequency not corresponding to the value of the minimum $\tau$, so revealing the difference between computing $1 / \tau=1 /\left\langle t_{i}\right\rangle$, and $v \propto\left\langle 1 / t_{i}\right\rangle$, with $t_{i}$ the TT for every translocation event [22]. As expected, and similarly to the previous case, the translocation probability shows that for low frequencies a fraction of the trials are not able to translocate. That is because the two values of the applied force are 0 and 0.6 , and no translocation occurs for the zero value of a constant force, while all the trajectories translocate at the 0.6 constant value. Thus, being the initial force randomly selected, $P_{i n}$ approaches 0.5 at low frequency. A study of the response of the system under constant force will be presented in the Summary and Conclusions section.

It is interesting to visualize the probability distribution function of the TTs (see Fig. 8) for three values of the frequency of the driving $v$. We can observe the three regimes: $v=10^{1}$ stands for the high frequency regime, where the TTs distribution is almost Gaussian, only slightly asymmetric. By decreasing the frequency, the distribution is more asymmetric and slightly moved toward smaller time values. The last panel corresponds to $v=10^{-2.5}$, a value in the low frequency region, where $P_{\text {in }} \simeq 0.5$. In this case the probability distribution is very wide and asymmetric. It is easy to understand this behavior: Typically, trials starting in the off state (minimum values of the driving force) will either move back into the cis region or translocate after some long time, while trials starting in the on state will more easily translocate, thus resulting in a low $\tau$. The global effect is a large value of $\tau$ for such a low frequency since it involves events with very high values of TTs.

\section{Scaling with $N$}

As we anticipated, the polymer size dependence mean TT scales with the same exponent $\alpha=1.6$ for the two driving forces studied in this paper. This feature points out that size scaling depends on polymer features and not on the pore actuation.

Figure 9 shows that all the curves plotted, having different polymer lengths, scale reasonably well for a large range of frequencies, with a RA minimum clearly visible for all the polymer lengths. Regarding the translocation probability, the

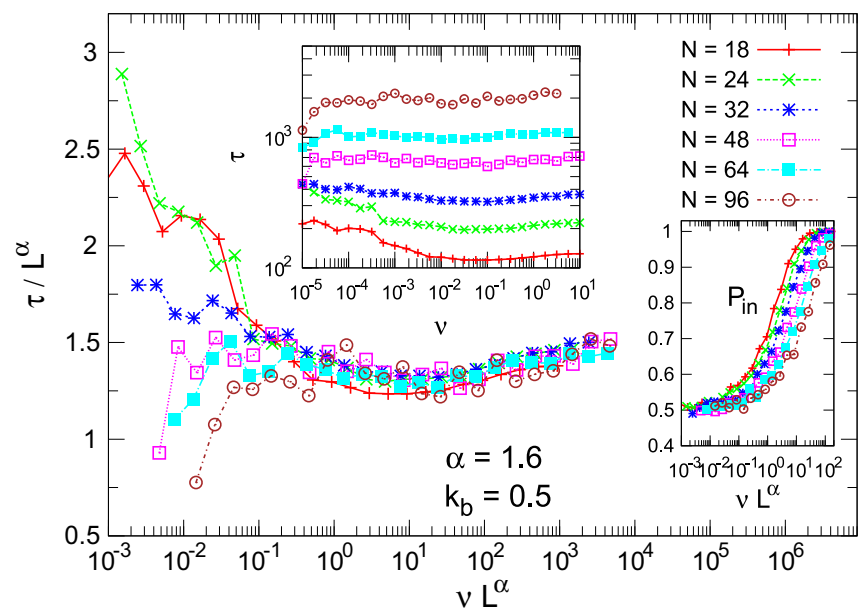

FIG. 9. (Color online) Scaled mean TT for different values of polymer length in the case of RTN.

increase of $P_{\text {in }}$ to 1 when increasing $v$ looks similar to that of the sinusoidal case.

The scaling appears confirmed, though not perfectly, for a wide range of high and intermediate frequencies. The absence of overlap at low frequency is due to the complex phenomenology in these simulations. In fact, at very low frequencies and large chains, the mean TT is affected by the presence of rare events with very high TTs. These events are difficult to capture in calculations. Moreover, some of the trajectories in these cases remain trapped up to the maximum simulation time we fixed in our simulations. The contribution of these rare events could change the TT in a nonobvious way.

As commented above, the TT, and so the scaling behavior, can, in general, be affected by specific situations as some choice of the parameters, the initial configuration, and finite size effects of the polymer $[8,46]$.

\section{The role of the bending parameter}

The presence of the minimum, as well as the low frequency saturation properties of $\tau$ under this driving, is strongly affected by the bending parameter $k_{b}$. Figure 10 shows the values of $\tau$ normalized by the high frequency limit $\tau_{H F L}$. Important qualitative differences at different $k_{b}$ values are observed. For high $k_{b}$ ( $k_{b} \geqslant 0.5$ in our simulations), $\tau$ is large at small frequencies, decreases when $v$ increases, reaches a minimum, and increases towards a constant high frequency value. At small $k_{b}$, however ( $k_{b} \leqslant 0.2$ in our simulations), $\tau$ is small at low $v$ and increases towards $\tau_{H F L}$ when increasing the frequency. The minimum in the intermediate frequency region remains always present. These differences are consequences of the elasticity of the chain. For low $k_{b}$ values, the chain is very soft ( $L_{p}$ is small), and the monomers can distribute closer to the entrance than in the stiff cases. Thus, the average distance from the trans side is lower for small $k_{b}$, and $\tau$ can decrease. On the contrary, for large $k_{b}$ (larger $L_{p}$ ), the polymer chain is more rigid and the distance from the entrance of the pore is higher than for small values, resulting in a larger TT mean.

The low $\tau$ value observed for small $k_{b}$ and small frequencies agrees with the results of Ikonen et al. [21]: For low $k_{b}$ we 


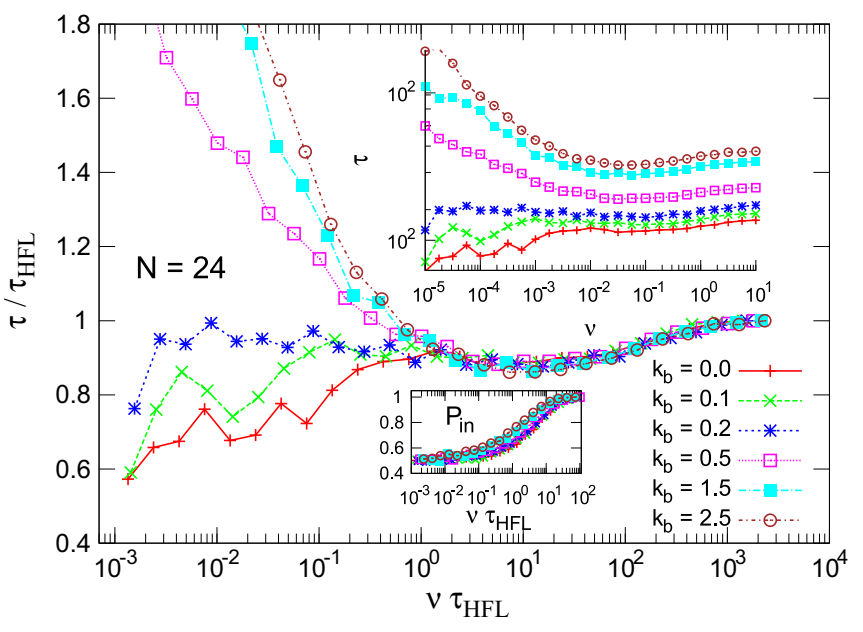

FIG. 10. (Color online) Normalized mean TT $\tau_{N}$ at different values of the bending parameter $k_{b}$ for the RTN driving. $N=24$.

recover the results of the FJC model. At high $k_{b}$, however, $\tau$ is large at small frequencies, as found for the 1D case [22].

Note also that the overall behavior of TT in Fig. 10 is similar to that in Fig. 9. This opens the possibility to make a correspondence between the length of the chain and the bending parameter by defining an effective length $L_{\text {eff }}=L / L_{p}$, which can be used as a scaled parameter in the translocation of a generic semiflexible polymer.

\section{SUMMARY AND CONCLUSIONS}

The study here presented concerns the translocation of a polymer chain through a repulsive, uniform pore membrane in a thermal fluctuating environment and under two different time dependent forces: the deterministic sinusoidal and the stochastic RTN. The two driving forces exhibit peculiar behaviors. The study is mainly devoted to the role of the rigidity parameter of a chain with excluded volume contributions, as well as the dependence of the mean TT on the polymer length. We notice a scaling behavior of the TTs with the polymer length according to a power law of the type $L^{\alpha}$, with $\alpha$ independent of both: the two different drivers used and their frequencies.

Some discrepancies are instead evident when considering different bending parameters of the chain. With the sinusoidal force, the qualitative behavior of the mean TT with the frequency is the same for all the $k_{b}$ values. Conversely, the RTN driving (of the same average and force variation as the sinusoidal) gives a different response at low frequencies and low bending values, reducing in those cases the TTs.

In order to better understand the different frequency limits, we have also performed a simulation for a polymer driven by a constant force $F_{c}$. The results of the calculated mean TT and $P_{i n}$ are shown in Fig. 11. As expected for low $F_{c}$ only a small fraction of the events translocate: The mean total force experienced by the polymer comes from the interaction with the membrane and pore and prevents the polymer translocation. This effect is more important for low bending, where a stronger constant driving $F_{c}$ is needed in order to get high translocation probability values. In our model, the high frequency limit basically corresponds to the

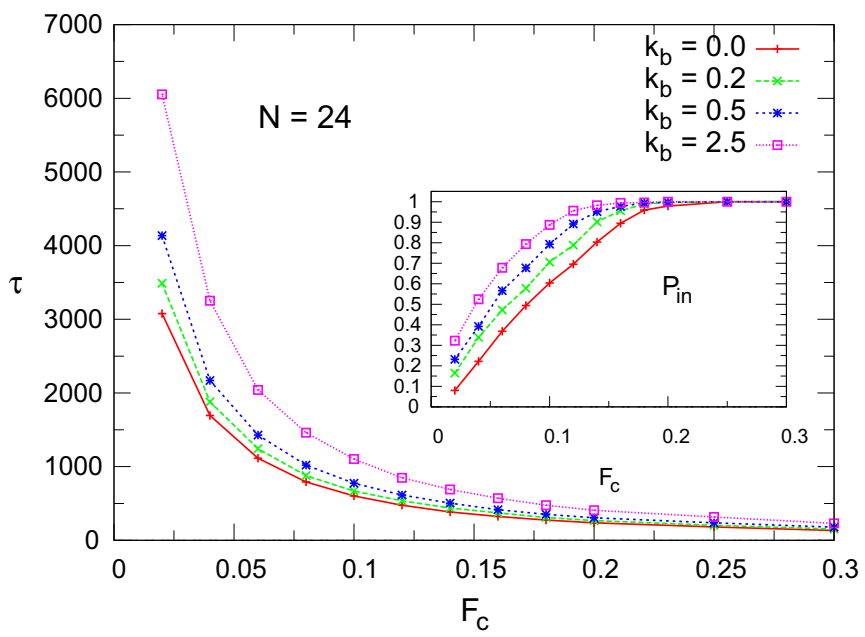

FIG. 11. (Color online) Mean TT as a function of the constant force $F_{c}$ for four values of the bending parameter $k_{b}$. (Inset) The corresponding translocation probability $P_{i n}$.

result obtained for $F_{c}=0.3$. Regarding the low frequency limit, for the sinusoidal case the average over different initial conditions results in an averaging over the results where the force $F_{c}$ changes between 0.0 and 0.6 , weighted with the proper probability translocation for each case. The RTN case averages over results with $F_{c}=0.0$ and $F_{c}=0.6$. To finish, we want to mention that, for the sinusoidal case, the minimum TT observed in Fig. 2 corresponds to $\tau \simeq 150$, a value very close to that obtained for constant $F_{c}=0.6$, showing an excellent efficiency of the driving force at that frequency value.

The analysis of the translocation probability of the polymer has been performed to help the understanding of the translocation dynamics. At low frequencies the dynamics is dominated by $P_{i n}$ smaller than 1 . However, at high frequency almost all the simulated chains translocate. The values of the translocation probability are smaller for low frequencies (approaching 0.8 in the sinusoidal case and 0.5 in the RTN one) and the transition to $P_{i n}=1$ occurs in a relatively narrow frequency interval.

The study of the translocation represents an interesting subject not only for the basic understanding of the translocation aspects of the long molecules, but also as a starting point in the construction and/or emulation of nanotechnological objects. In this regard, the present work can be further developed in two directions: On the one hand by the usage of more realistic pores than a rigid and uniform channel; On the other hand by enlarging the driving to other forms, as the molecular motor able to use the ATP molecules for its functioning that has been successfully modeled in previous studies $[23,24]$.

\section{ACKNOWLEDGMENTS}

This work is supported by the Spanish Project No. FIS201125167, cofinanced by FEDER funds, and EPSRC Project No. GALE EP/K020633/1. We also thank the support of the Aragón Government to FENOL group. 
[1] J. J. Kasianowicz, E. Brandin, D. Branton, and D. W. Deamer, Proc. Natl. Acad. Sci. USA 93, 13770 (1996).

[2] M. Zwolak and M. Di Ventra, Rev. Mod. Phys. 80, 141 (2008).

[3] G. F. Schneider et al., Nano Lett. 10, 3163 (2010).

[4] J. Li, D. Stein, C. McMullan, D. Brantin, and D. W. Deamer, Nature (London) 412, 166 (2001).

[5] M. Mickler, E. Schleiff, and T. Hugel, ChemPhysChem 9, 1503 (2008).

[6] E. B. Starikov, D. Henning, H. Yamada, R. Gutiérrez, B. Norden, and G. Cuniberti, Biophys. Rev. Lett. 04, 209 (2009).

[7] P. Rowghanian and A. Y. Grosberg, J. Phys. Chem. B 115, 14127 (2011).

[8] T. Ikonen, A. Bhattacharya, T. Ala-Nissila, and W. Sung, Phys. Rev. E 85, 051803 (2012).

[9] A. Meller, J. Phys.: Condens. Matter 15, R581 (2003).

[10] A. Milchev, J. Phys.: Condens. Matter 23, 103101 (2011).

[11] N. Pizzolato, A. Fiasconaro, D. Persano Adorno, and B. Spagnolo, Phys. Biol. 7, 034001 (2010).

[12] N. Pizzolato, A. Fiasconaro, D. Persano Adorno, and B. Spagnolo, J. Chem. Phys. 138, 054902 (2013).

[13] W. Sung and P. J. Park, Phys. Rev. Lett. 77, 783 (1996).

[14] M. T. Downton, M. J. Zuckermann, E. M. Craig, M. Plischke, and H. Linke, Phys. Rev. E 73, 011909 (2006); E. M. Craig, M. J. Zuckermann, and H. Linke, ibid. 73, 051106 (2006).

[15] I. Kosztin and K. Schulten, Phys. Rev. Lett. 93, 238102 (2004).

[16] D. E. Smith, S. J. Tans, S. B. Smith, S. Grimes, D. L. Anderson, and C. Bustamante, Nature (London) 413, 748 (2001).

[17] J. R. Moffitt, Y. R. Chemla, K. Aathavan, S. Grimes, P. J. Jardine, D. L. Anderson, and C. Bustamante, Nature (London) 457, 446 (2009).

[18] A. Fiasconaro, J. J. Mazo, and F. Falo, Phys. Rev. E 82, 031803 (2010).

[19] J. A. Cohen, A. Chaudhuri, and R. Golestanian, Phys. Rev. Lett. 107, 238102 (2011).

[20] J. A. Cohen, A. Chaudhuri, and R. Golestanian, J. Chem. Phys. 137, 204911 (2012).

[21] T. Ikonen, J. Shin, W. Sung, and T. Ala-Nissila, J. Chem. Phys. 136, 205104 (2012).

[22] A. Fiasconaro, J. J. Mazo, and F. Falo, J. Stat. Mech. (2011) P11002.
[23] A. Fiasconaro, J. J. Mazo, and F. Falo, New J. Phys. 14, 023004 (2012).

[24] R. Pérez-Carrasco, A. Fiasconaro, F. Falo, and J. M. Sancho, Phys. Rev. E 87, 032721 (2013).

[25] P. E. J. Rouse, J. Chem. Phys. 21, 1272 (1953).

[26] T. Ikonen, A. Bhattacharya, T. Ala-Nissila, and W. Sung, J. Chem. Phys. 137, 085101 (2012).

[27] G. Sigalov, J. Comer, G. Timp, and A. Aksimentiev, Nano Lett. 8, 56 (2008).

[28] H. W. de Haan and G. W. Slater, Phys. Rev. Lett. 110, 048101 (2013).

[29] D. Rodríguez-Larrea and H. Bayley, Nat. Nanothech. 8, 288 (2013).

[30] J. Han, S. W. Turner, and H. G. Craighead, Phys. Rev. Lett. 83, 1688 (1999).

[31] B. Luan, H. Peng, S. Polonsky, S. Rossnagel, G. Stolovitzky, and G. Martyna, Phys. Rev. Lett. 104, 238103 (2010).

[32] M. M. Millonas and D. R. Chialvo, Phys. Rev. Lett. 76, 550 (1996).

[33] A. Kargol and K. Kabza, Phys. Biol. 5, 026003 (2008).

[34] M. Fyta, S. Melchionna, and S. Sucii, Polym. Phys. 49, 985 (2011).

[35] C. R. Doering and J. C. Gadoua, Phys. Rev. Lett. 69, 2318 (1992).

[36] M. Bier and R. D. Astumian, Phys. Rev. Lett. 71, 1649 (1993).

[37] A. Fiasconaro and B. Spagnolo, Phys. Rev. E 83, 041122 (2011).

[38] A. Fiasconaro and F. Falo, Phys. Rev. E 86, 032902 (2012).

[39] P. M. Suhonen, K. Kaski, and R. P. Linna, Phys. Rev. E 90, 042702 (2014)

[40] P. Rowghanian and A. Y. Grosberg, Phys. Rev. E 87, 042722 (2013).

[41] A. Yu. Grosberg, S. Nechaev, M. Tamm, and O. Vasilyev, Phys. Rev. Lett. 96, 228105 (2006).

[42] K. Luo, T. Ala-Nissila, S. C. Ying, and R. Metzler, Europhys. Lett. 88, 68006 (2009).

[43] H.-P. Hsu, W. Paul, and K. Binder, Polymer Science Series C 55, 39 (2013).

[44] R. Adhikari and A. Bhattacharya, J. Chem. Phys. 138, 204909 (2013).

[45] Y. Kantor and M. Kardar, Phys. Rev. E 69, 021806 (2004).

[46] T. Ikonen, A. Bhattacharya, T. Ala-Nissila, and W. Sung, Europhys. Lett. 103, 38001 (2013). 